\title{
Lifetime of Triplet Photosensitizers in Aerosol Using Time-Resolved Photoelectric Activity
}

Ephraim Woods III, Oliver T. Harris, William E. Leiter, Nora E. Burner, Pomaa Ofosuhene, Alexandra Krez, Mark A. Hilton, and Kathleen A. Burke

Colgate University, Department of Chemistry, 13 Oak Drive, Hamilton, NY 13346

\section{Supporting Information}

13 pages, 8 tables

Contents

1. Estimating Particle Phase Concentrations

2. Description of DFT Calculations

3. DFT Results

IC $\left(\mathrm{S}_{0}\right)$

$\mathrm{IC}\left(\mathrm{T}_{1}\right)$

$\mathrm{IC}^{+}$

IC dimer \#1

IC dimer \#2

$\mathrm{AQ} 2 \mathrm{~S}\left(\mathrm{~S}_{0}\right)$

AQ2S $\left(T_{1}\right)$

AQ2S neutral radical

Table of electron binding energies
Page

S2

$\mathrm{S} 2$

S4

S5

S6

S7

S8

S9

$\mathrm{S} 10$

$\mathrm{S} 11$

S12 


\section{Estimating Particle Phase Concentrations}

We estimate the concentration of solutes as a function of $\mathrm{RH}$ in these predominantly $\mathrm{NaCl}$ aerosol particles using the E-AIM Model III. ${ }^{1-2}$ As a first estimate, we carry out the calculation with only $\mathrm{NaCl}$, using the option to suppress the formation of $\mathrm{NaCl}(\mathrm{s})$ in the supersaturated regime $(<75 \% \mathrm{RH})$. The concentration of the photosensitizer is less than $1 \%$ of the concentration of chloride, so the associated error is less than that which arises from uncertainty in our measured $\mathrm{RH}$. The latter uncertainty, $\pm 2 \%$, leads to an approximately 5 to $15 \%$ error in the concentration of solute, depending on the RH. To calculate the concentration of photosensitizer, we scale the $\mathrm{NaCl}$ concentration by the photosensitizer to $\mathrm{NaCl}$ ratio in the atomizer solution. For example, at $65 \%$ $\mathrm{RH}$, the $\mathrm{NaCl}$ concentration is $8.3 \mathrm{~mol} / \mathrm{kg}$ and the model-calculated density is $1.25 \mathrm{~g} / \mathrm{cm}^{3}$. Using these values, the $\mathrm{NaCl}$ molarity is $7.0 \mathrm{~mol} / \mathrm{L}$. For a typical solution, where the photosensitizer: $\mathrm{NaCl}$ mole ratio is 0.01 , the concentration of photosensitizer is $0.070 \mathrm{~mol} / \mathrm{L}$.

Repeating this approach with species that are included in the E-AIM model, such as nitrate, results in discrepancies of approximately $5 \%$ between the estimate and the model-calculated value for the relatively small concentrations in this study. For an aqueous aerosol with composition $\left(\mathrm{NaCl}: \mathrm{NaNO}_{3}=100: 1\right)$, E-AIM predicts $\left[\mathrm{NO}_{3}{ }^{-}\right]=0.074 \mathrm{~mol} / \mathrm{L}$. The approximate procedure outlined above, for which the identity of the solute does not matter, yields a value of $0.070 \mathrm{~mol} / \mathrm{L}$.

\section{Description of DFT Calculations}

We used the Gaussian $16^{3}$ software to carry out DFT calculations. We first optimized geometries and calculated vibrational frequencies and thermochemical data at the B3LYP/6$311 \mathrm{G}(\mathrm{d})$ level of theory. We calculated single point electronic energies with this optimized geometry at the B3LYP/6-311++G(2df,2p) level of theory both for isolated molecules and using 
the $\mathrm{SMD}^{4}$ implicit solvent model with the solvent set to water. We used empirical dispersion corrections $\left(\mathrm{GD}^{5}\right)$ at both stages of the calculation.

In the tables that follow, $\mathrm{E}_{0}$ is the electronic energy, $\mathrm{ZPE}$ is the zero-point energy, $\Delta \mathrm{E}_{\text {therm }}$ is the thermal correction to the energy, $\Delta \mathrm{H}_{\text {therm }}$ is the thermal correction to the enthalpy, and $\Delta \mathrm{G}_{\text {therm }}$ is the thermal correction to the free energy. All the thermal corrections assume a temperature of 298 K. The electron binding energies shown in Figure 2 of the main article and tabulated in Table S8 are the adiabatic ionization energies at $0 \mathrm{~K}$ for each species, calculated as the difference in $\mathrm{E}_{0}($ water $)+\mathrm{ZPE}$ for $\mathrm{IC}$ and $\mathrm{IC}^{+}$or AQ2S and AQ2S-neutral radical. 
3. DFT Results

IC $\left(\mathrm{S}_{0}\right)$

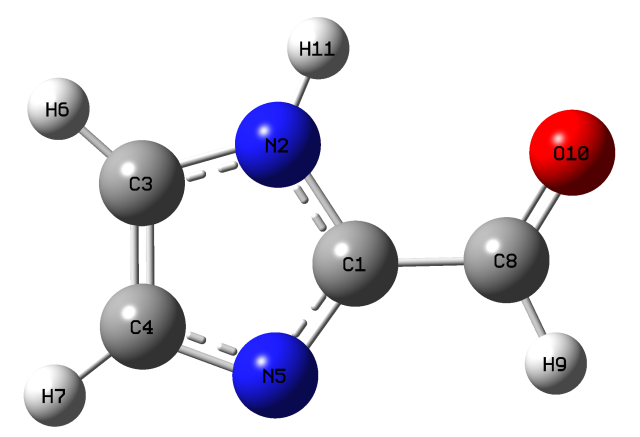

\begin{tabular}{llll|ll}
\hline \hline \multicolumn{6}{l}{ Table S1. Calculated geometry and thermochemistry for IC $\left(\mathrm{S}_{0}\right)$} \\
\hline \multicolumn{7}{l}{ Coordinates (standard orientation) } & \multicolumn{2}{l}{ Thermochemistry } \\
\hline Center & $\mathrm{X}$ & $\mathrm{Y}$ & $\mathrm{Z}$ & \multicolumn{2}{l}{ Energy / Hartree } \\
\hline $\mathrm{C} 1$ & -0.233570 & -0.278671 & -0.000014 & $\mathrm{E}_{0}$ (gas) & -339.672918479 \\
$\mathrm{~N} 2$ & 0.295938 & 0.983623 & 0.000086 & $\mathrm{E}_{0}($ water) & -339.688190740 \\
$\mathrm{C} 3$ & 1.652930 & 0.855158 & -0.000138 & $\mathrm{ZPE}$ & 0.080685 \\
$\mathrm{C} 4$ & 1.892780 & -0.507026 & -0.000180 & $\Delta \mathrm{E}_{\text {therm }}$ & 0.086171 \\
$\mathrm{~N} 5$ & 0.719580 & -1.198946 & -0.000073 & $\Delta \mathrm{H}_{\text {therm }}$ & 0.087116 \\
$\mathrm{H} 6$ & 2.316214 & 1.704221 & -0.000204 & $\Delta \mathrm{G}_{\text {therm }}$ & 0.051333 \\
H7 & 2.848171 & -1.008452 & -0.000277 & & \\
C8 & -1.674517 & -0.507240 & 0.000030 & & \\
H9 & -1.964198 & -1.573556 & 0.000023 & & \\
O10 & -2.484949 & 0.397781 & 0.000240 & & \\
H11 & -0.254954 & 1.829479 & 0.000264 & &
\end{tabular}


IC $\left(\mathrm{T}_{1}\right)$

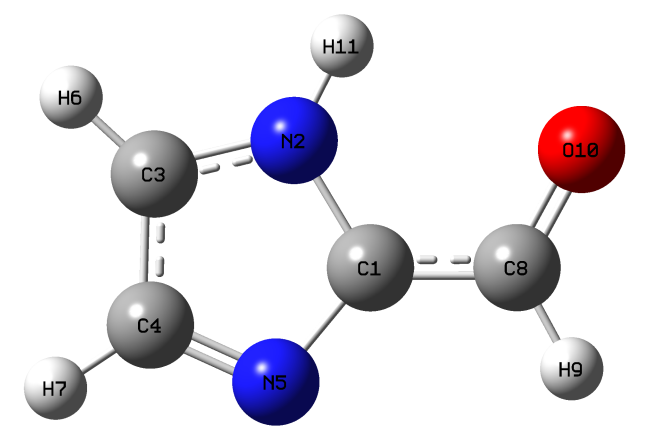

\begin{tabular}{|c|c|c|c|c|c|}
\hline \multicolumn{4}{|c|}{ Coordinates (standard orientation) } & \multicolumn{2}{|c|}{ Thermochemistry } \\
\hline Center & $\mathrm{X}$ & $\mathrm{Y}$ & $\mathrm{Z}$ & & Energy / Hartree \\
\hline $\mathrm{C} 1$ & -0.265474 & -0.326166 & -0.000066 & $\mathrm{E}_{0}$ (gas) & -339.565743724 \\
\hline N2 & 0.226253 & 0.997325 & -0.000268 & $\mathrm{E}_{0}$ (water) & -339.586346180 \\
\hline $\mathrm{C} 3$ & 1.590563 & 0.926914 & -0.000048 & ZPE & 0.075695 \\
\hline $\mathrm{C} 4$ & 1.890346 & -0.478451 & -0.000087 & $\Delta \mathrm{E}_{\text {therm }}$ & 0.081284 \\
\hline N5 & 0.816771 & -1.229294 & -0.000179 & $\Delta \mathrm{H}_{\text {therm }}$ & 0.082229 \\
\hline H6 & 2.235007 & 1.790220 & -0.000023 & $\Delta \mathrm{G}_{\text {therm }}$ & 0.045360 \\
\hline H7 & 2.886113 & -0.903594 & -0.000171 & & \\
\hline $\mathrm{C} 8$ & -1.635142 & -0.579592 & 0.000356 & & \\
\hline H9 & -1.971032 & -1.620728 & 0.000092 & & \\
\hline $\mathrm{O} 10$ & -2.443529 & 0.412935 & 0.000235 & & \\
\hline H11 & -0.265474 & -0.326166 & -0.000066 & & \\
\hline
\end{tabular}


$\mathrm{IC}^{+}$

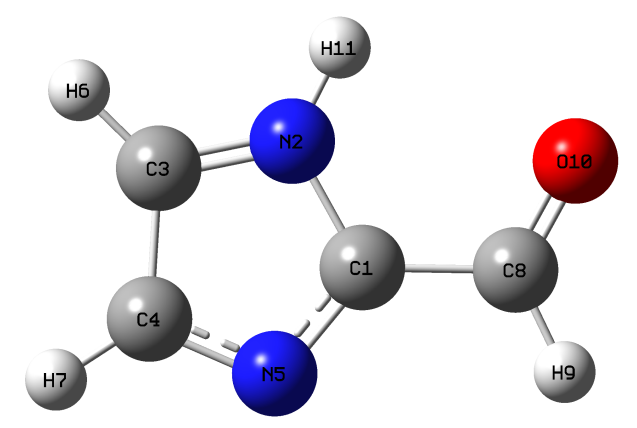

Table S3. Calculated geometry and thermochemistry for $\mathrm{IC}^{+}$

\begin{tabular}{llll|ll}
\hline \multicolumn{4}{l|}{ Coordinates (standard orientation) } & \multicolumn{2}{l}{ Thermochemistry } \\
\hline Center & $\mathrm{X}$ & $\mathrm{Y}$ & $\mathrm{Z}$ & & Energy / Hartree \\
\hline $\mathrm{C} 1$ & -0.227388 & -0.315670 & 0.000022 & $\mathrm{E}_{0}$ (gas) & -339.337274989 \\
$\mathrm{~N} 2$ & 0.273853 & 0.993337 & 0.000134 & $\mathrm{E}_{0}$ (water) & -339.448858958 \\
$\mathrm{C} 3$ & 1.593964 & 0.913534 & -0.000214 & $\mathrm{ZPE}$ & 0.078896 \\
$\mathrm{C} 4$ & 1.888381 & -0.521236 & -0.000186 & $\Delta \mathrm{E}_{\text {therm }}$ & 0.084686 \\
$\mathrm{~N} 5$ & 0.760012 & -1.215810 & -0.000039 & $\Delta \mathrm{H}_{\text {therm }}$ & 0.085631 \\
$\mathrm{H} 6$ & 2.253219 & 1.771261 & -0.000313 & $\Delta \mathrm{G}_{\text {therm }}$ & 0.048452 \\
H7 & 2.869635 & -0.977472 & -0.000319 & & \\
C8 & -1.696627 & -0.546601 & 0.000240 & & \\
H9 & -2.028552 & -1.593791 & -0.000170 & & \\
O10 & -2.421244 & 0.418812 & 0.000082 & & \\
H11 & -0.311385 & 1.826663 & 0.000313 & &
\end{tabular}


IC dimer \#1

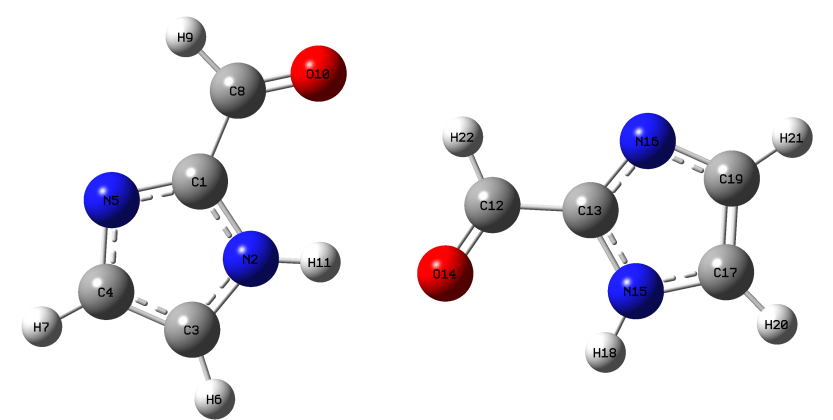

\begin{tabular}{|c|c|c|c|c|c|}
\hline \multicolumn{4}{|c|}{ Coordinates (standard orientation) } & \multicolumn{2}{|c|}{ Thermochemistry } \\
\hline Center & $\mathrm{X}$ & $\mathrm{Y}$ & Z & & Energy / Hartree \\
\hline $\mathrm{C} 1$ & -3.057764 & 0.501913 & 0.000267 & $\mathrm{E}_{0}$ (gas) & -679.361971 \\
\hline $\mathrm{N} 2$ & -2.304449 & -0.640621 & -0.001201 & $\mathrm{E}_{0}$ (water) & -679.384749 \\
\hline C3 & -3.172403 & -1.685994 & 0.000568 & ZPE & 0.162943 \\
\hline $\mathrm{C} 4$ & -4.441092 & -1.127747 & 0.002930 & $\Delta \mathrm{E}_{\text {therm }}$ & 0.175688 \\
\hline N5 & -4.359557 & 0.227484 & 0.002899 & $\Delta \mathrm{H}_{\text {therm }}$ & 0.176632 \\
\hline H6 & -2.838194 & -2.710673 & 0.000014 & $\Delta \mathrm{G}_{\text {therm }}$ & 0.120300 \\
\hline $\mathrm{H} 7$ & -5.390294 & -1.641399 & 0.004747 & & \\
\hline $\mathrm{C} 8$ & -2.496253 & 1.842802 & -0.001111 & & \\
\hline H9 & -3.264958 & 2.637028 & 0.000902 & & \\
\hline $\mathrm{O} 10$ & -1.304924 & 2.095114 & -0.004119 & & \\
\hline H11 & -1.279496 & -0.688893 & -0.002854 & & \\
\hline $\mathrm{C} 12$ & 1.261969 & 0.153900 & -0.000469 & & \\
\hline C13 & 2.709915 & 0.078426 & 0.000473 & & \\
\hline $\mathrm{O} 14$ & 0.565468 & -0.856613 & -0.003504 & & \\
\hline N15 & 3.379672 & -1.117703 & -0.002025 & & \\
\hline N16 & 3.556252 & 1.099842 & 0.003681 & & \\
\hline $\mathrm{C} 17$ & 4.711095 & -0.837154 & -0.000229 & & \\
\hline H18 & 2.931640 & -2.022137 & -0.004637 & & \\
\hline C19 & 4.796729 & 0.545441 & 0.003217 & & \\
\hline $\mathrm{H} 20$ & 5.466038 & -1.606003 & -0.001540 & & \\
\hline $\mathrm{H} 21$ & 5.689992 & 1.150523 & 0.005397 & & \\
\hline $\mathrm{H} 22$ & 0.824324 & 1.161017 & 0.001598 & & \\
\hline
\end{tabular}


IC dimer \#2

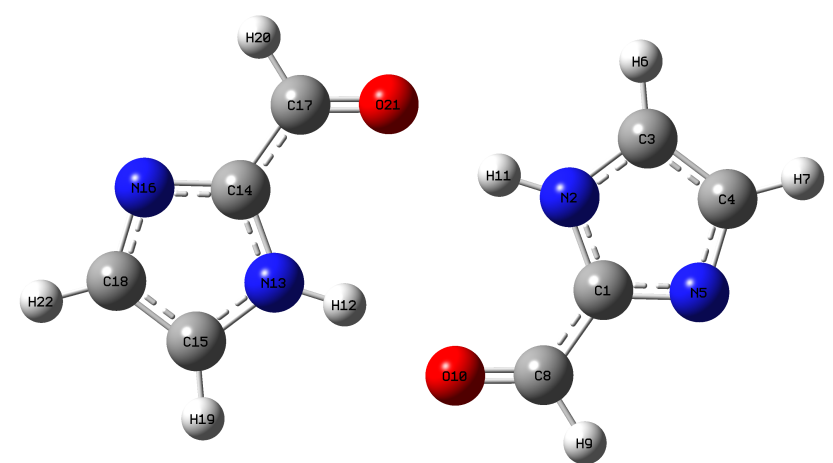

Table S5. Calculated geometry and thermochemistry for IC dimer \#1

\begin{tabular}{|c|c|c|c|c|c|}
\hline \multicolumn{4}{|c|}{ Coordinates (standard orientation) } & \multicolumn{2}{|c|}{ Thermochemistry } \\
\hline Center & $\mathrm{X}$ & $\mathrm{Y}$ & Z & & Energy / Hartree \\
\hline $\mathrm{C} 1$ & 2.5181830 & -0.6953720 & -0.0002520 & $\mathrm{E}_{0}$ (gas) & -679.372993 \\
\hline $\mathrm{N} 2$ & 2.0502750 & 0.5922320 & -0.0005570 & $\mathrm{E}_{0}$ (water) & -679.388985 \\
\hline $\mathrm{C} 3$ & 3.1331650 & 1.4062580 & -0.0005640 & $\mathrm{ZPE}$ & 0.163448 \\
\hline $\mathrm{C} 4$ & 4.2420160 & 0.5690510 & -0.0000700 & $\Delta \mathrm{E}_{\text {therm }}$ & 0.175855 \\
\hline N5 & 3.8514180 & -0.7268540 & -0.0000200 & $\Delta \mathrm{H}_{\text {therm }}$ & 0.176799 \\
\hline H6 & 3.0397510 & 2.4799410 & -0.0008430 & $\Delta \mathrm{G}_{\text {therm }}$ & 0.122110 \\
\hline $\mathrm{H} 7$ & 5.2835830 & 0.8512870 & 0.0000970 & & \\
\hline $\mathrm{C} 8$ & 1.6869210 & -1.8752470 & -0.0001950 & & \\
\hline H9 & 2.2632210 & -2.8152050 & -0.0000490 & & \\
\hline $\mathrm{O} 10$ & 0.4620990 & -1.8833890 & -0.0004940 & & \\
\hline H11 & 1.0730890 & 0.8988230 & -0.0008390 & & \\
\hline $\mathrm{H} 12$ & -1.0735420 & -0.8991380 & 0.0000010 & & \\
\hline N13 & -2.0505970 & -0.5923350 & 0.0003320 & & \\
\hline $\mathrm{C} 14$ & -2.5180860 & 0.6954730 & 0.0002690 & & \\
\hline $\mathrm{C} 15$ & -3.1337370 & -1.4059820 & 0.0007130 & & \\
\hline N16 & -3.8512920 & 0.7273470 & 0.0009490 & & \\
\hline $\mathrm{C} 17$ & -1.6864250 & 1.8749770 & -0.0003250 & & \\
\hline $\mathrm{C} 18$ & -4.2423200 & -0.5684080 & 0.0010940 & & \\
\hline H19 & -3.0407310 & -2.4797070 & 0.0007340 & & \\
\hline $\mathrm{H} 20$ & -2.2623360 & 2.8151840 & -0.0001120 & & \\
\hline $\mathrm{O} 21$ & -0.4615980 & 1.8823700 & -0.0006900 & & \\
\hline $\mathrm{H} 22$ & -5.2839830 & -0.8502600 & 0.0015350 & & \\
\hline
\end{tabular}




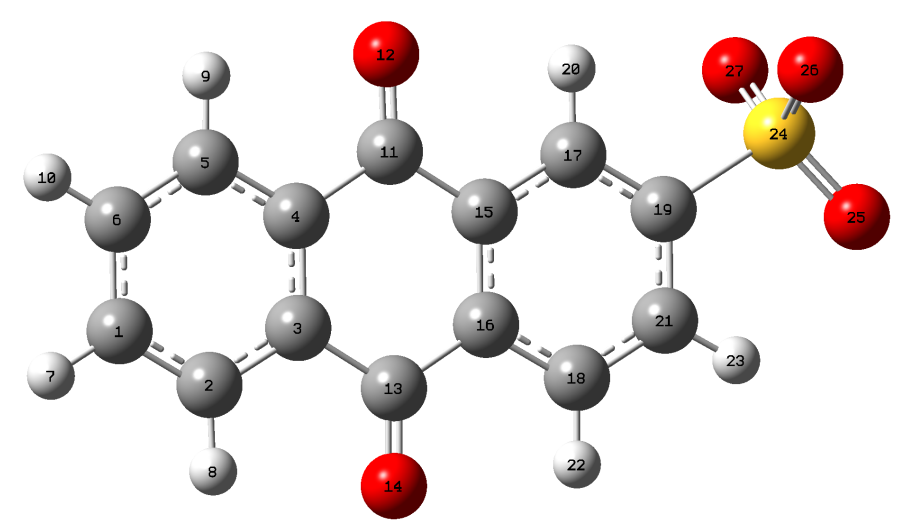

\begin{tabular}{|c|c|c|c|c|c|}
\hline \multicolumn{4}{|c|}{ Coordinates (standard orientation) } & \multicolumn{2}{|c|}{ Thermochemistry } \\
\hline Center & $\mathrm{X}$ & $\mathrm{Y}$ & $\mathrm{Z}$ & & Energy / Hartree \\
\hline $\mathrm{C} 1$ & 5.593225 & -0.315378 & 0.050308 & $\mathrm{E}_{0}$ (gas) & -1312.471478 \\
\hline $\mathrm{C} 2$ & 4.662981 & 0.716167 & 0.065260 & $\mathrm{E}_{0}$ (water) & -1312.569121 \\
\hline $\mathrm{C} 3$ & 3.294500 & 0.432374 & 0.029152 & $\mathrm{ZPE}$ & 0.181807 \\
\hline $\mathrm{C} 4$ & 2.862427 & -0.901478 & -0.022378 & $\Delta \mathrm{E}_{\text {therm }}$ & 0.197153 \\
\hline C6 & 3.804589 & -1.933997 & -0.037122 & $\Delta \mathrm{H}_{\text {therm }}$ & 0.198097 \\
\hline C6 & 5.162781 & -1.644268 & -0.001031 & $\Delta \mathrm{G}_{\text {therm }}$ & 0.135871 \\
\hline $\mathrm{H7}$ & 6.654809 & -0.088531 & 0.078531 & & \\
\hline H8 & 4.967994 & 1.755489 & 0.104794 & & \\
\hline H9 & 3.442417 & -2.954771 & -0.077170 & & \\
\hline H10 & 5.889461 & -2.451060 & -0.012744 & & \\
\hline $\mathrm{C} 11$ & 1.403336 & -1.248502 & -0.062301 & & \\
\hline $\mathrm{O} 12$ & 1.053935 & -2.417137 & -0.106817 & & \\
\hline $\mathrm{C} 13$ & 2.315065 & 1.566111 & 0.046426 & & \\
\hline $\mathrm{O} 14$ & 2.714579 & 2.722494 & 0.091627 & & \\
\hline $\mathrm{C} 15$ & 0.435054 & -0.122965 & -0.045143 & & \\
\hline $\mathrm{C} 16$ & 0.877838 & 1.220856 & 0.006927 & & \\
\hline $\mathrm{C} 17$ & -0.930904 & -0.402262 & -0.081109 & & \\
\hline $\mathrm{C} 18$ & -0.069195 & 2.248420 & 0.021383 & & \\
\hline C19 & -1.865120 & 0.633860 & -0.066153 & & \\
\hline $\mathrm{H} 20$ & -1.260858 & -1.433136 & -0.120808 & & \\
\hline $\mathrm{C} 21$ & -1.428460 & 1.958572 & -0.014800 & & \\
\hline $\mathrm{H} 22$ & 0.284479 & 3.272639 & 0.061411 & & \\
\hline $\mathrm{H} 23$ & -2.178198 & 2.741295 & -0.004318 & & \\
\hline $\mathrm{S} 24$ & -3.653715 & 0.247678 & -0.114045 & & \\
\hline $\mathrm{O} 25$ & -4.306188 & 1.576278 & -0.083219 & & \\
\hline $\mathrm{O} 26$ & -3.853350 & -0.571853 & 1.101585 & & \\
\hline $\mathrm{O} 27$ & -3.804320 & -0.489994 & -1.387782 & & \\
\hline
\end{tabular}


AQS $\left(T_{1}\right)$

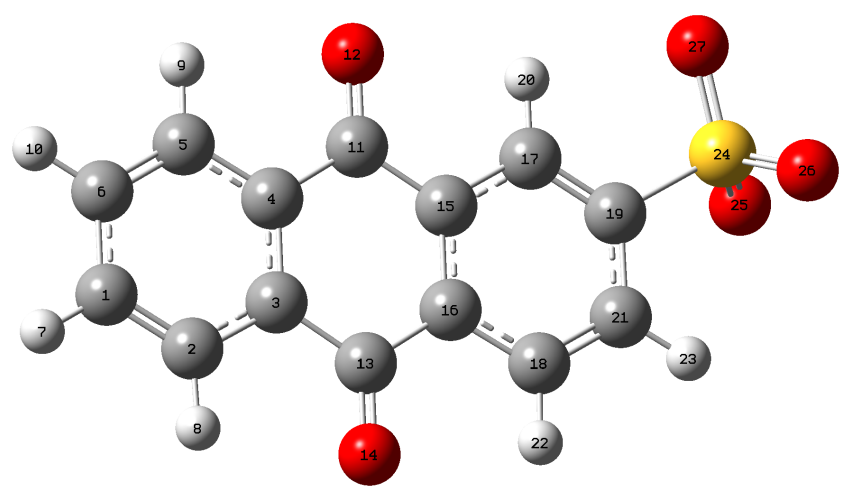

\begin{tabular}{|c|c|c|c|c|c|}
\hline \multicolumn{4}{|c|}{ Coordinates (standard orientation) } & \multicolumn{2}{|c|}{ Thermochemistry } \\
\hline Center & $\mathrm{X}$ & $\mathrm{Y}$ & $\mathrm{Z}$ & & Energy / Hartree \\
\hline $\mathrm{C} 1$ & 5.254372 & 0.041617 & 0.002657 & $\mathrm{E}_{0}(\mathrm{gas})$ & -1312.388052 \\
\hline $\mathrm{C} 2$ & 4.231897 & 0.968140 & 0.001049 & $\mathrm{E}_{0}$ (water) & -1312.470290 \\
\hline $\mathrm{C} 3$ & 2.882109 & 0.563623 & -0.000388 & $\mathrm{ZPE}$ & 0.177280 \\
\hline $\mathrm{C} 4$ & 2.580519 & -0.822440 & 0.000905 & $\Delta \mathrm{E}_{\text {therm }}$ & 0.193018 \\
\hline C6 & 3.641198 & -1.758155 & 0.001497 & $\Delta \mathrm{H}_{\text {therm }}$ & 0.193962 \\
\hline C6 & 4.953247 & -1.336670 & 0.002674 & $\Delta \mathrm{G}_{\text {therm }}$ & 0.131726 \\
\hline $\mathrm{H} 7$ & 6.289527 & 0.371447 & 0.003703 & & \\
\hline $\mathrm{H} 8$ & 4.428548 & 2.034748 & 0.000055 & & \\
\hline H9 & 3.388363 & -2.812658 & 0.001492 & & \\
\hline H10 & 5.757132 & -2.067757 & 0.003766 & & \\
\hline $\mathrm{C} 11$ & 1.203557 & -1.298088 & 0.001480 & & \\
\hline $\mathrm{O} 12$ & 0.938048 & -2.533966 & -0.004095 & & \\
\hline $\mathrm{C} 13$ & 1.831362 & 1.589624 & -0.004693 & & \\
\hline $\mathrm{O} 14$ & 2.098107 & 2.806500 & -0.006532 & & \\
\hline $\mathrm{C} 15$ & 0.150292 & -0.293245 & 0.000701 & & \\
\hline $\mathrm{C} 16$ & 0.451695 & 1.092763 & -0.002804 & & \\
\hline $\mathrm{C} 17$ & -1.201651 & -0.710667 & -0.000466 & & \\
\hline $\mathrm{C} 18$ & -0.610658 & 2.021581 & 0.001188 & & \\
\hline C19 & -2.209473 & 0.224494 & -0.001219 & & \\
\hline $\mathrm{H} 20$ & -1.428144 & -1.769434 & 0.000879 & & \\
\hline $\mathrm{C} 21$ & -1.923281 & 1.608385 & 0.000773 & & \\
\hline $\mathrm{H} 22$ & -0.350606 & 3.074230 & 0.002771 & & \\
\hline $\mathrm{H} 23$ & -2.733275 & 2.328606 & 0.005066 & & \\
\hline S24 & -3.929019 & -0.297995 & 0.003012 & & \\
\hline $\mathrm{O} 25$ & -4.539510 & 0.234229 & -1.245105 & & \\
\hline $\mathrm{O} 26$ & -4.545744 & 0.307085 & 1.211111 & & \\
\hline $\mathrm{O} 27$ & -3.938194 & -1.780978 & 0.033865 & & \\
\hline
\end{tabular}


AQS-neutral radical

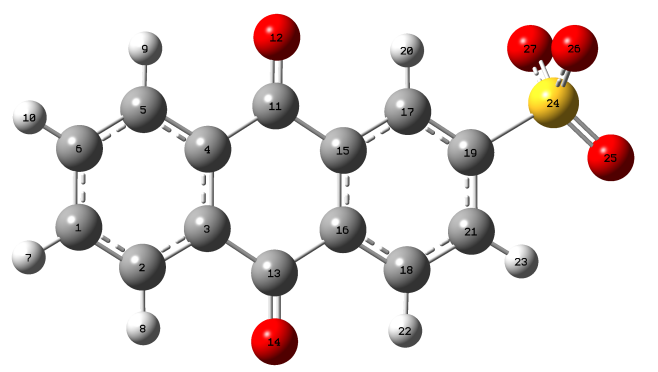

Table S7. Calculated geometry and thermochemistry for AQS-neutral

\begin{tabular}{|c|c|c|c|c|c|}
\hline & \multicolumn{3}{|c|}{ Coordinates (standard orientation) } & \multicolumn{2}{|c|}{ Thermochemistry } \\
\hline Center & $\mathrm{X}$ & $\mathrm{Y}$ & $\mathrm{Z}$ & & Energy / Hartree \\
\hline $\mathrm{C} 1$ & 5.267447 & 0.013787 & -0.000389 & E0(gas) & -1312.295057 \\
\hline $\mathrm{C} 2$ & 4.248635 & 0.958294 & -0.000200 & $\mathrm{E}_{0}$ (water) & -1312.311133 \\
\hline $\mathrm{C} 3$ & 2.911842 & 0.549239 & 0.000008 & ZPE & 0.180670 \\
\hline $\mathrm{C} 4$ & 2.602769 & -0.823765 & 0.000056 & $\Delta \mathrm{E}_{\text {therm }}$ & 0.196395 \\
\hline C6 & 3.634935 & -1.766252 & -0.000107 & $\Delta \mathrm{H}_{\text {therm }}$ & 0.197339 \\
\hline C6 & 4.960357 & -1.349555 & -0.000345 & $\Delta \mathrm{G}_{\text {therm }}$ & 0.135095 \\
\hline $\mathrm{H} 7$ & 6.303133 & 0.336519 & -0.000570 & & \\
\hline $\mathrm{H} 8$ & 4.464189 & 2.020123 & -0.000205 & & \\
\hline H9 & 3.374324 & -2.817929 & -0.000036 & & \\
\hline H10 & 5.757668 & -2.085140 & -0.000496 & & \\
\hline $\mathrm{C} 11$ & 1.192564 & -1.301987 & 0.000305 & & \\
\hline $\mathrm{O} 12$ & 0.914340 & -2.486872 & 0.000566 & & \\
\hline $\mathrm{C} 13$ & 1.843034 & 1.585449 & 0.000184 & & \\
\hline $\mathrm{O} 14$ & 2.099812 & 2.775588 & 0.000361 & & \\
\hline $\mathrm{C} 15$ & 0.116351 & -0.261543 & 0.000182 & & \\
\hline $\mathrm{C} 16$ & 0.421987 & 1.109669 & 0.000119 & & \\
\hline $\mathrm{C} 17$ & -1.214483 & -0.677736 & 0.000145 & & \\
\hline $\mathrm{C} 18$ & -0.605428 & 2.055868 & 0.000003 & & \\
\hline C19 & -2.219763 & 0.282315 & 0.000006 & & \\
\hline $\mathrm{H} 20$ & -1.431589 & -1.739216 & 0.000224 & & \\
\hline $\mathrm{C} 21$ & -1.933218 & 1.649111 & -0.000046 & & \\
\hline $\mathrm{H} 22$ & -0.343336 & 3.106888 & -0.000011 & & \\
\hline $\mathrm{H} 23$ & -2.740515 & 2.370920 & -0.000157 & & \\
\hline S24 & -3.927436 & -0.248675 & -0.000123 & & \\
\hline $\mathrm{O} 25$ & -4.774050 & 0.932086 & -0.000145 & & \\
\hline $\mathrm{O} 26$ & -4.114334 & -1.194839 & 1.154645 & & \\
\hline $\mathrm{O} 27$ & -4.114153 & -1.194805 & -1.154965 & & \\
\hline
\end{tabular}




\begin{tabular}{|c|c|c|c|c|}
\hline Species & $\begin{array}{l}\text { Eo(water)+ZPE } \\
\text { (Hartree) }\end{array}$ & $\begin{array}{l}\left(\mathrm{E}_{0}\left(\text { water)+ZPE) } \mathrm{IC}^{+}\right.\right. \\
\text {or AQ2S-neutral rad. } \\
\text { (Hartree) }\end{array}$ & IP/Hartree & $\mathrm{IP} / \mathrm{eV}$ \\
\hline $\mathrm{IC}\left(\mathrm{S}_{0}\right)$ & -339.6881907 & -339.44886 & 0.238 & 6.46 \\
\hline $\mathrm{IC}\left(\mathrm{T}_{1}\right)$ & -339.58635 & -339.44886 & 0.141 & 3.83 \\
\hline $\mathrm{AQ} 2 \mathrm{~S}\left(\mathrm{~S}_{0}\right)$ & -1312.5691 & -1312.3111 & 0.257 & 6.99 \\
\hline AQ2S $\left(T_{1}\right)$ & -1312.4703 & -1312.3111 & 0.1623 & 4.42 \\
\hline
\end{tabular}




\section{References}

(S1) Clegg, S. L.; Brimblecombe, P.; Wexler, A. S., Thermodynamic Model of the System $\mathrm{H}^{+}-$ $\mathrm{Nh}_{4}{ }^{+}-\mathrm{Na}^{+}-\mathrm{SO}_{4}{ }^{2-}-\mathrm{NO}_{3}{ }^{-}-\mathrm{Cl}^{-}-\mathrm{H}_{2} \mathrm{O}$ at 298.15 K. J. Phys. Chem. A 1998, 102, 2155-2171.

(S2) Clegg, S. L.; Brimblecombe, P.; Wexler, A. S. Extented Aim Thermodynamics Model. http://www.aim.env.uea.ac.uk/aim/aim.php (accessed March 2020).

(S3) Frisch, M. J.; Trucks, G. W.; Schlegel, H. B.; Scuseria, G. E.; Robb, M. A.; Cheeseman, J. R.; Scalmani, G.; Barone, V.; Petersson, G. A.; Nakatsuji, H.; Li, X.; Caricato, M.; Marenich, A. V.; Bloino, J.; Janesko, B. G.; Gomperts, R.; Mennucci, B.; Hratchian, H. P.; Ortiz, J. V.; Izmaylov, A. F.; Sonnenberg, J. L.; Williams; Ding, F.; Lipparini, F.; Egidi, F.; Goings, J.; Peng, B.; Petrone, A.; Henderson, T.; Ranasinghe, D.; Zakrzewski, V. G.; Gao, J.; Rega, N.; Zheng, G.; Liang, W.; Hada, M.; Ehara, M.; Toyota, K.; Fukuda, R.; Hasegawa, J.; Ishida, M.; Nakajima, T.; Honda, Y.; Kitao, O.; Nakai, H.; Vreven, T.; Throssell, K.; Montgomery Jr., J. A.; Peralta, J. E.; Ogliaro, F.; Bearpark, M. J.; Heyd, J. J.; Brothers, E. N.; Kudin, K. N.; Staroverov, V. N.; Keith, T. A.; Kobayashi, R.; Normand, J.; Raghavachari, K.; Rendell, A. P.; Burant, J. C.; Iyengar, S. S.; Tomasi, J.; Cossi, M.; Millam, J. M.; Klene, M.; Adamo, C.; Cammi, R.; Ochterski, J. W.; Martin, R. L.; Morokuma, K.; Farkas, O.; Foresman, J. B.; Fox, D. J. Gaussian 16 Rev. C.01, Wallingford, CT, 2016.

(S4) Marenich, A. V.; Cramer, C. J.; Truhlar, D. G., Universal Solvation Model Based on Solute Electron Density and on a Continuum Model of the Solvent Defined by the Bulk Dielectric Constant and Atomic Surface Tensions. J. Phys. Chem. B 2009, 113, 6378-6396.

(S5) Grimme, S.; Antony, J.; Ehrlich, S.; Krieg, H., A Consistent and Accurate Ab Initio Parametrization of Density Functional Dispersion Correction (Dft-D) for the 94 Elements H-Pu. J. Chem. Phys. 2010, 132, 154104. 